\title{
Efecto de un programa de ejercicio terapéutico en la mejora funcional y prevención de lesiones de rodilla en el fútbol sala femenino semiprofesional
}

\author{
Loreto Ferrández Laliena ${ }^{1}$, Rocío Sánchez Rodríguez ${ }^{1}$, Lucía Vicente Pina ${ }^{1}$, María \\ Orosia Lucha López ${ }^{1}$, Sofía Monti Ballano ${ }^{1}$, Jaime Corral de Toro ${ }^{1}$, Daniel Fernández \\ de Landa Nicolau ${ }^{1}$, José Miguel Tricás Moreno ${ }^{1}$ \\ ${ }^{1}$ Afiliación: Unidad de Investigación de Fisioterapia (UIF) \\ Instituto de Investigación en Ingeniería de Aragón (I3A) \\ Universidad de Zaragoza, Mariano Esquillor s/n, 50018, Zaragoza, Spain.
}

Tel. +34-976762707, e-mail: loretousk@gmail.com

\section{Resumen}

Ensayo clínico controlado diseñado con el objetivo de disminuir la elevada prevalencia de lesiones de ligamento cruzado anterior en el fútbol femenino. Se implementó un programa específico de prevención de lesiones, obteniéndose mejoras significativas en la capacidad funcional y en la incidencia de lesiones en las jugadoras.

\section{Introducción}

En fútbol femenino, la estructura con mayor incidencia de lesiones es el ligamento cruzado anterior (LCA) siendo la lesión que más tiempo de baja deportiva provoca(1). El mecanismo lesional habitual es non contact, por un fallo en el patrón de activación neuromuscular en situaciones de estabilización. Al deberse a la adquisición de patrones de movimiento patológicos se considera prevenible mediante la reversión de los factores de riesgo. Existen numerosos estudios que han analizado los factores modificables que inciden en el patrón motor, biomecánicos y neuromusculares; el desplazamiento medial del eje de carga en acciones de estabilización dinámica; la inhibición de la musculatura glútea; el incremento del valgo dinámico (VD), por el bostezo anteromedial y el impacto lateral articular; el desequilibrio en el ratio de reclutamiento isquiotibiales/cuádriceps (H/Q) y retardo en la activación de la musculatura isquiotibial por fatiga. Todavía quedan áreas sujetas a controversia que articulan las hipótesis de investigación actuales como la inhibición o fatiga precoz de los isquiotibiales $(\mathrm{H})$ y del glúteo mayor (GMax), la implicación del valor del ratio H/Q y el $\mathrm{VD}$ en los planos frontal y transversal intrínseco articular, en la lesión de LCA. No obstante, las últimas investigaciones al respecto son estudios teóricos. No existen estudios que hayan trasladado la valoración de la efectividad de programas de prevención integrales a jugadoras de fútbol femenino semiprofesionales. Es por ello que este estudio se propuso implementar un programa específico de prevención de lesiones, fundamentado en la estimulación y corrección del patrón de reclutamiento de la musculatura de GMax y $\mathrm{H}$, el reequilibrio del ratio $\mathrm{H} / \mathrm{Q}$ y la estimulación de la cocontracción previa al impacto o desequilibrio y el feedback propioceptivo para la reestabilización específica en el plano frontal/transversal, y valorar su efectividad en la reducción de incidencia de lesiones en la extremidad inferior, especialmente lesiones de LCA de rodilla y en la mejora de la capacidad funcional del miembro inferior en jugadoras de fútbol sala.

\section{Métodos}

Para conseguir los objetivos, se diseñó un ensayo clínico controlado. Se reclutaron como muestra 22 jugadoras federadas en las plantillas de dos equipos

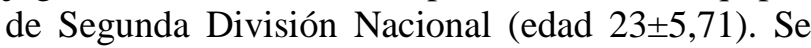
dividieron en dos grupos, experimental (GE) y control (GC). Se excluyeron aquellas jugadoras que presentaban lesiones en el momento del inicio del estudio. En el GE se implementó un programa específico de prevención de lesiones de 7 meses de duración. Se diseñaron sesiones con una periodización semanal que incluían el entrenamiento de fuerza específica, y de fuerza compensatoria, estimulación de la recuperación y asistencia fisioterápica, de 30 minutos de duración y realización previa a los entrenamientos. Se progresaba en los requerimientos de control biomecánico y neuromuscular demandados a la jugadora. Mientras, el GC continuó con una rutina independiente de prevención de lesiones basada en el programa FIFA 11+.

Se evaluaron variables biomecánicas y se registró la incidencia lesional, pre y post intervención. El registro de las variables biomecánicas se llevó a cabo mediante videograbado con un móvil Samsung S8 y posterior análisis con Kinovea 8.5.1. El análisis de datos se hizo con el programa IBM SPSS 
Statistics 20.0, realizando un análisis descriptivo, comparativo de muestras independientes y relacionadas.

\section{Resultados}

Se alcanzó significación estadística en la mejora de la capacidad funcional del GE respecto al GC $(\mathrm{p}=0,000)$. Además de una mejora significativa interindividual en el GE en el VD, tiempo de estabilización (TTS) y desplazamiento medial de rodilla (DMR) en ambas piernas, dominante (PD) y no dominante (PND) $(\mathrm{p}=0,001)$, entre otras. El registro de incidencia lesional mostró un valor de ratio muy inferior en el GE frente al GC, 2,247 y 5,213 lesiones por 1000 hexp respectivamente.

\section{Discusión}

El objetivo de este estudio era valorar los efectos de un programa de prevención de lesiones específico en la mejora de la capacidad funcional e incidencia lesional en jugadoras de fútbol sala. Los resultados muestran que se ha reducido significativamente el incremento de $\mathrm{VD}$, principal factor de riesgo de rotura de LCA, en el GE en ambas piernas y además los valores son significativamente menores que en el GC. El GE pasó de presentar valores de riesgo de lesión de LCA, incremento de VD superior a $15^{\circ}(2)$, a presentar un valor final de $5,07^{\circ}$ en la PD y $4,71^{\circ}$ en la PND. Mientras que el GC alcanzó valores de riesgo de $18,5^{\circ}$ en la $\mathrm{PD}$ y $21,83^{\circ}$ en la PND. Los estudios más recientes sobre biomecánica del VD explican que la acción conjunta de un bostezo medial e impacto lateral aumentados, incrementan el VD y por tanto el riesgo de lesión de LCA(3). En este estudio se demuestra que las jugadoras del GE han disminuido significativamente el DMR en ambas piernas, disminuyendo la medialización del eje de carga y por tanto, disminuyendo el bostezo medial articular. Mientras que el GC no alcanzó mejora significativa y además aumentó notablemente el valor de DMR en la PND, pierna de estabilización en desequilibrio monopodal, mecanismo habitual de lesión de LCA(4). Los resultados indican que se ha reducido significativamente el TTS en el GE en ambas piernas, alcanzando un valor de $24,79 \mathrm{~ms}$ en la PD y 24,50 ms en la PND. Según demostraron Ishida et al. en su estudio, el momento en el que se produce la lesión de LCA es 50 ms después del efecto del desequilibrio(5), valor que coincide con el TTS de la PND en el GC, lo cual refleja un aumento del riesgo de lesión. Además la activación de $\mathrm{H}$ comienza en mujeres de manera retardada, $20 \mathrm{~ms}$ después de asumir el efecto de la fuerza desequilibrante(6). Por tanto el en GE, al presentar un TTS de $24 \mathrm{~ms}$, se deduce que consigue una activación precoz de $\mathrm{H}$ suficiente para frenar el torque del Q, a diferencia de lo que ocurre en el GC.

El GE muestra al final del estudio un valor de ratio de incidencia de lesiones muy inferior al del GC y a los valores referencia de la bibliografía. Ruiz-Pérez et al.(1) alcanzaron un valor de 6,75 lesiones por 1000 hexp un su estudio prospectivo de tres temporadas en un equipo de Segunda División Nacional femenina de fútbol sala.

\section{Conclusión}

Los resultados de este estudio muestran como la implementación de un programa de entrenamiento específico ha permitido mejorar la capacidad funcional de las jugadoras de fútbol sala. Se han mejorado especialmente las conductas de estabilización biomecánica de riesgo para la patogénesis de la lesión de LCA como son el VD, el TTS y el DMR. Además se constata la función preventiva del programa diseñado en la reducción de la incidencia de lesiones.

[1].RUIZ-PÉREZ, I., LÓPEZ-VALENCIANO, A., JIMÉNEZLOAISA, A., ELVIRA, J.L., DE STE CROIX, M., AYALA, F.. Injury incidence, characteristics and burden among female sub-elite futsal players: a prospective study with three-year follow-up. PeerJ. 2019. Available from: doi:10.7717/peerj.7989

[2]. KROSSHAUG, T., NAKAMAE, A., BODEN, B.P., ENGEBRETSEN, L., SMITH, G., SLAUTERBECK, J.R., HEWETT, T.E., BAHR, R. Mechanisms of anterior cruciate ligament injury in basketball: Video analysis of 39 cases. American Journal of Sports Medicine. 2007, 35(3), 359-367. Available from: doi:10.1177/0363546506293899

[3]. NAVACCHIA, A., BATES, N.A., SCHILATY, N.D., KRYCH, J.A., HEWETT, T.E.. Knee Abduction and Internal Rotation Moments Increase ACL Force During Landing Through the Posterior Slope of the Tibia. Journal of Orthopaedic Research. 2019, 37(8), 1730-1742. Available from: doi:10.1002/jor.24313

[4].LARRUSKAIN, J., LEKUE, J.A., DIAZ, N., ODRIOZOLA, A., GIL, S.M.. A comparison of injuries in elite male and female football players: A five-season prospective study. Scandinavian Journal of Medicine and Science in Sport. 2018, 28(1), 237-245. Available form:10.1111/sms.12860

[5]. ISHIDA, T., KOSHINO, Y., YAMANAKA, M., UENO, R., TANIGUCHI, S., SAMUKAWA, M., SAITO, H., MATSUMOTO, H., AOKI, Y., TOHYAMA, H. The effects of a subsequent jump on the knee abduction angle during the early landing phase. BMC Musculoskeletal Disorders. 2018, 19(1), 1-9. Available from: doi:10.1186/s12891-0182291-4

[6].BEHRENS, M., MAU-MOELLER, A., WASSERMANN, F., BRUHN, S. Effect of Fatigue on Hamstring Reflex Responses and Posterior-Anterior Tibial Translation in Men and Women. PLoS ONE [online]. 2013, 8(2). Availbale from: doi:10.1371/journal.pone.0056988 